\title{
Estudo retrospectivo de abscessos hepáticos em bovinos abatidos em um frigorífico paulista
}

\author{
Retrospective study of liver abscesses in beef cattle slaughtered in a Brazilian abattoir \\ Thales dos Anjos de Faria VECHIATO'; Wagner MASCHIO²; Luiz Cesar BOM²; Paulo Domingos
LOPES $^{2}$; Enrico Lippi ORTOLANI ${ }^{1}$
}

${ }^{1}$ Departamento de Clínica Médica da Faculdade de Medicina Veterinária e Zootecnia da

Universidade de São Paulo, São Paulo - SP, Brasil

${ }^{2}$ Fiscal Federal Agropecuário, Médico Veterinário SIF 337, DISPOA/DAS/MAPA

\begin{abstract}
Resumo
O presente estudo se baseou em levantamento retrospectivo obtidos de bovinos abatidos em frigorífico paulista. Foram utilizados os registros do Serviço de Inspeção Federal de ocorrência de alterações hepáticas em 1.568 .821 bovinos (85\% machos e 15\% fêmeas), proveniente de seis Estados (SP, MS, PR, GO, MT e MG) durante os anos de 2002 a 2006. Consideraram-se os animais abatidos no último trimestre de cada ano como terminados em confinamento, sendo os demais criados continuamente em regime extensivo. Os abscessos hepáticos $(1,60 \%)$ foram a segunda mais frequente alteração hepática após a teleangectasia (1,67\%). A frequência desses abscessos foi maior em bovinos confinados (2,54\%) que nos criados extensivamente $(1,28 \%)$ e em fêmeas $(1,85 \%)$ que em machos $(1,56 \%)$. O confinamento aumenta o fator de risco (FR) de surgimento de abscessos hepáticos na ordem de 2,01 vezes. Maior frequência de abscessos foi registrada em bovinos oriundos do Paraná, em ambos os sistemas de terminação.
\end{abstract}

Palavras-chave: Abscesso hepático. Confinamento. Bovino.

\begin{abstract}
A retrospective survey was carried out in cattle slaughtered in a private abattoir in the State of Sao Paulo, Brazil. The survey was based on the reports done by the Federal Inspection Service of the liver condemnations among 1,568,821 cattle (85\% steers; 15\% cows and heifers) from six different Brazilian states during 2002 and 2006. Feedlot cattle were mostly slaughtered in the last trimester of each year, while the cattle bred extensively were in the remaining months. The abscesses (1.60\%) were the second highest cause of liver condemnation after telangiectasis (1.67\%). The frequency of liver abscesses were higher in feedlot cattle (2.54\%) than in cattle raised exclusively on pasture (1.28\%), female (1.85\%) than male $(1.56 \%)$. Feedlot increased the outcome (odds ratio OD $=2.01 \mathrm{x}$ ) of liver abscess. The highest frequency of liver abscess was detected in feedlot and extensive farming cattle system from the Parana state.
\end{abstract}

Keywords: Liver abscess. Feedlot. Cattle.

\section{Introdução}

Sistemas de confinamentos de bovinos são cada vez mais comuns e visam aumentar o ganho de peso, melhorar a conversão alimentar, acabamento de gordura na carcaça, qualidade da carne e taxa de desfrute. Para almejar estes objetivos produtivos no confinamento, a utilização de dietas ricas em concentrados energéticos tem se intensificado. No entanto, se por um lado estas dietas podem trazem enormes vantagens, por outro, se forem mal utilizadas podem predispor o surgimento de problemas metabólicos, tais como acidose ruminal, ruminites, abscessos hepáticos e outras enfermidades, entre elas as broncopneumonias ${ }^{1}$.
Embora os aspectos epidemiológicos dessas afecções metabólicas e infecções supracitadas tenham sido muito bem estudados nos países com pecuária de corte mais desenvolvida, destacando-se os Estados Unidos, Austrália, Canadá entre outros, poucos e incipientes estudos nacionais foram realizados até

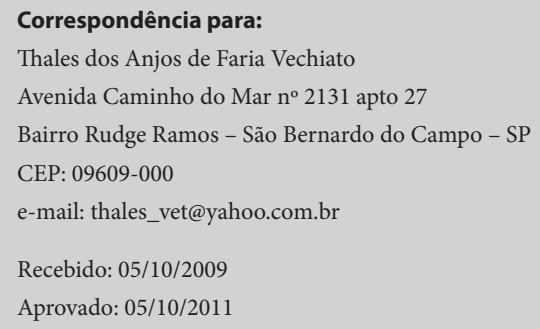


o momento, em especial quanto à frequência de abscessos hepáticos ${ }^{2,3}$.

De acordo com a literatura, a principal causa dos abscessos hepáticos encontrados em bovinos abatidos nos matadouros e frigoríficos é proveniente de quadros prévios de ruminites, desencadeados inicialmente por distúrbios digestivos, em especial, pela acidose ruminal ${ }^{3,4,5,6,7,8,9,10,11}$.

Após se instalar o quadro de ruminite, as barreiras físicas de defesa estão comprometidas devido à lesão originada, o que facilita a entrada de bactérias ruminais, em especial, as do gênero Fusobacterium necrophorum. Essas bactérias após invadirem a mucosa ruminal, atingem a circulação sanguínea e, através do sistema circulatório porta, chegam ao fígado, ocasionando infecção e formação de abscessos hepáticos ${ }^{12}$.

Os abscessos hepáticos causam uma redução em até $11 \%$ no ganho de peso diário, redução de 9,7\% na eficiência alimentar e diminuição na taxa de crescimento de 5,85\% a $12,7 \%$ nos animais acometidos, o que pode ocasionar um grande impacto econômico para os pecuaristas e demais elos ligados à cadeia da

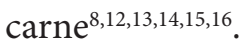

Normalmente os abscessos hepáticos são formados por bactérias anaeróbicas, destacando as do gênero Fusobaterium necrophorum, isoladas entre 71 a 95\% dos casos, seguido pelas do gênero Corynebacterium pyogenes com 20 a 35\% e 6\% pelas do gênero Sthaphylococcus sp. e Streptococcus $\mathrm{sp}^{10,12}$.

Os abscessos hepáticos podem ocorrer independentemente da faixa etária, sexo ou raça. Estes apresentam uma incidência de $1-2 \%$ em animais provenientes de pastagens, mas quando arraçoados com dietas ricas em concentrado energético têm seus valores aumentados para $90-95 \%$ e, com frequência em torno de 12 a $32 \%{ }^{11}$.

Devido à dificuldade em se estabelecer um diagnóstico desta enfermidade, os abscessos são comumente diagnosticados e encontrados no exame post-mortem durante o abate de bovinos. Estes fígados são examinados pelos métodos de inspeção e palpação por agentes do serviço federal de inspeção (SIF) e condenado caso surja alterações macroscópicas. Essas alterações e causas de condenação podem ser ocasionadas por quadros de teleangiectasia, esteatose, congestão, hidatidose, fasciolose, perihepatite e abscessos hepáticos ${ }^{2,3,17}$.

Em experimento conduzido em alguns frigoríficos do Estado de Minas Gerais, foi observado condenação de 50.405 (8,29\%) em 607.346 fígados analisados. Dentre as enfermidades encontradas, a teleangiectasia foi responsável pela maior $(25,12 \%)$ causa de condenação (12.663 fígados), seguido por abscessos hepáticos $(24,32 \%)$, perihepatite $(13,04 \%)$, hidatidose $(4,65 \%)$, congestão $(2,23 \%)$ e outras causas não classificados frente sua etiologia $(30,58 \%)^{2}$.

A frequência dos abscessos hepáticos entre os anos de 1992 a 2002 foi de 3,10\%, ou seja, 4.786 casos dentro do total de 154.379 bovinos abatidos. Concluiu-se que ocorra uma maior frequência $(19,29 \%)$ no período de entressafra, quando comparado ao período de safra $(18,15 \%)$, com concentração principalmente na estação de inverno $(10,41 \%)^{18}$. Porém no Brasil, nenhum estudo abrangente relatou a presença de enfermidades hepáticas, em especial abscessos hepáticos, quanto sua predisposição sexual e tipo de terminação.

Deste modo, o presente estudo objetivou avaliar a frequência das enfermidades hepáticas em bovinos abatidos em um frigorífico paulista, quanto à predisposição sexual e de tipo de terminação das mesmas.

\section{Material e Método}

Foi realizado um levantamento retrospectivo e prospectivo em um frigorífico paulista, localizado no extremo oeste do Estado de São Paulo, no qual se coletou dados quanto à frequência de enfermidades hepáticas condenadas (abscesso hepático, teleangiectasia, perihepatite, esteatose, congestão, hidatidose e fasciolose) em bovinos abatidos entre os anos de 2002 
a 2006. Os dados foram provenientes da inspeção sanitária individual dos animais e dos seus respectivos fígados. Os exames de inspeção foram realizados pelos médicos veterinários e/ou técnicos especializados do Ministério da Agricultura, Pecuária e Abastecimento (MAPA).

Neste trabalho foi convencionado que os bovinos abatidos durante os meses de outubro, novembro e dezembro, de cada ano, foram considerados predominantemente como terminados em regime de confinamento, enquanto que os abatidos nos demais meses como animais terminados em regime extensivo. Tal convenção foi baseada no depoimento dos médicos veterinários do SIF, do supracitado frigorífico, os quais afirmaram que a maioria dos animais abatidos no último trimestre do ano era proveniente de confinamentos especializados, boitéis ou mesmo propriedades que realizavam cria, recria e engorda, sendo esta última em regime de confinamento.

Todos os dados analisados foram testados quanto sua predisposição em relação ao tipo de terminação e frequência sexual, utilizando-se para tal o teste de duas proporções com aproximação normal descrito por Sampaio ${ }^{19}$, no qual se comparam paralelamente resultados obtidos das frequências proporcionais entre os dados estudados. Para se verificar a influência de uma variável (condição de criação, sexo, enfermidade etc.) e cálculo de fator de risco (FR) em relação ao surgimento de uma determinada enfermidade, foi realizado o cálculo do fator de risco $(\mathrm{FR})^{20}$. Todos os dados tabulados foram submetidos a testes estatísticos processados por meio do programa estatístico computadorizado ${ }^{21}$.

\section{Resultados}

Dos 1.568.821 animais avaliados no período estudado, a frequência de condenação de fígados foi de 4,37\% (68.536) (Tabela 1) em bovinos abatidos originados de diferentes estados federativos (São Paulo, Mato Grosso do Sul, Paraná, Goiás, Mato Grosso e Minas Gerais). Os machos representaram $85 \%$ e as fêmeas 15\% do total abatido entre os anos de 2002 a $2006(\mathrm{P}<0,001)$.

A tabela 2 mostra a frequência das lesões hepáticas condenadas pelos agentes do SIF, na qual são distribuídas de acordo com o tipo de terminação na qual se procurou avaliar a quantidade, em números globais e porcentagem das mesmas. Maior percentual de bovinos confinados teve seus fígados condenados por abscesso hepático e congestão, ocorrendo o inverso na teleangiectasia e hidatidose $(\mathrm{P}<0,0001)$. Considerando-se todas as causas de condenação hepática

Tabela 1 - Enfermidades hepáticas condenadas pelos agentes do SIF entre os anos de 2002 a 2006 - São Paulo - 2009

\begin{tabular}{cccc}
\hline $\begin{array}{c}\text { Enfermidades } \\
\text { hepáticas }\end{array}$ & Total em números & $\begin{array}{c}\text { Percentual entre os } \\
\text { fígados condenados } \\
(\%)\end{array}$ & $\begin{array}{c}\text { Ocorrência entre } \\
\text { bovinos abatidos (\%) }\end{array}$ \\
\hline Teleangiectasia & 26.259 & 38,34 & $1,67^{\mathrm{a}}$ \\
Abscesso hepático & 25.216 & 36,77 & $1,60^{\mathrm{b}}$ \\
Perihepatite & 12.774 & 18,64 & $0,81^{\mathrm{c}}$ \\
Congestão & 2.113 & 3,08 & $0,13^{\mathrm{d}}$ \\
Hidatidose & 1.071 & 1,56 & $0,069^{\mathrm{e}}$ \\
Esteatose & 992 & 1,45 & $0,063^{\mathrm{e}}$ \\
Fasciolose & 111 & 0,16 & $0,007^{\mathrm{f}}$ \\
\hline Total & $\mathbf{6 8 . 5 3 6}$ & $\mathbf{1 0 0}$ & \\
\hline
\end{tabular}

Letras diversas entre as linhas diferem entre si $(\mathrm{P}<0,0001)$ 
Tabela 2 - Influência do tipo de terminação sobre as alterações hepáticas que levaram a suas condenações no período estudado e fator de risco (FR) - São Paulo - 2009

\begin{tabular}{|c|c|c|c|c|c|}
\hline \multirow{3}{*}{ Enfermidade } & \multicolumn{4}{|c|}{ Tipo de terminação } & \multirow{3}{*}{ Fator de risco (FR) } \\
\hline & \multicolumn{2}{|c|}{ Fígados condenados (n) } & \multirow{2}{*}{ Extensivo (\%) } & \multirow{2}{*}{ Confinado (\%) } & \\
\hline & Extensivo & Confinado & & & \\
\hline Abscesso hepático & 14.964 & 10.252 & $1,28^{\mathrm{b}}$ & $2,54^{\mathrm{a}}$ & 2,01 \\
\hline Teleangiectasia & 19.909 & 6.350 & $1,71^{\mathrm{a}}$ & $1,58^{\mathrm{b}}$ & 0,92 \\
\hline Perihepatite & 9.351 & 3.423 & $0,80^{\mathrm{a}}$ & $0,85^{\mathrm{a}}$ & 1,06 \\
\hline Congestão & 1.641 & 472 & $0,14^{\mathrm{b}}$ & $0,18^{\mathrm{a}}$ & 0,83 \\
\hline Esteatose & 745 & 247 & $0,06^{\mathrm{a}}$ & $0,06^{\mathrm{a}}$ & 0,96 \\
\hline Hidatidose & 860 & 211 & $0,07^{\mathrm{a}}$ & $0,05^{\mathrm{b}}$ & 0,76 \\
\hline Fasciolose & 81 & 30 & $0,007^{\mathrm{a}}$ & $0,007^{\mathrm{a}}$ & 1,07 \\
\hline
\end{tabular}

Letras diferentes entre as colunas diferem entre si $(\mathrm{P}<0,0001)$

em relação ao número global de bovinos, segundo forma de terminação, constatou-se que os submetidos ao confinamento apresentaram maior percentual (5,27\%), em relação aos terminados extensivamente $(4,07 \%)(\mathrm{P}<0,0001)$. Realizou-se o fator de risco $(\mathrm{FR})$ em relação aos tipos de terminação sobre a frequência de fígados condenados por diferentes enfermidades e condições. Destaca-se entre os resultados o maior risco $(\mathrm{FR}=2,01)$ que o confinamento pode trazer no surgimento de abscessos hepáticos, em relação à terminação extensiva.

Quanto à frequência global dos os abscessos hepáticos, os mesmos foram 1,97\% no ano de 2002, 2,08\% em $2003,1,62 \%$ em $2004,1,22 \%$ em 2005 e 1,18\% no ano de 2006 (Tabela 3). Estes foram mais frequentes em todos os anos estudados em bovinos terminados em confinamento em relação aos terminados extensivamente, $2,54 \%$ e $1,28 \%(\mathrm{P}<0,0001)$, respectivamente. De modo geral, as fêmeas apresentaram maior percentual $(1,85 \%)$ de abscessos hepáticos que os machos $(1,56 \%)$, independente do tipo de terminação (Tabela 4). Em relação aos valores referentes ao fator de risco do tipo de terminação e do sexo em predispor o surgimento de abscessos hepáticos, o confinamento aumentou o índice em relação à terminação extensiva
$(\mathrm{FR}=2,01)$, tanto em machos como em fêmeas submetidos aos dois regimes. Parece não existir influência sexual, quando não se leva em consideração o tipo de terminação $(F R=0,84)$.

A tabela 5 mostra os valores referentes ao fator de risco do tipo de terminação e do sexo em predispor o surgimento de abscessos hepáticos. O confinamento aumentou o índice em relação à terminação extensiva, tanto em machos como em fêmeas submetidos aos dois regimes. Parece não existir influência sexual, quando não se leva em consideração o tipo de terminação.

\section{Discussão}

O número de bovinos abatidos em um Frigorífico paulista (1.568.821 cabeças) no Estado de São Paulo, durante os anos de 2002 a 2006, e acompanhados no presente levantamento retrospectivo, representa entre $1,13 \%$ a $1,41 \%$ dos animais sacrificados e inspecionados desta espécie no Brasil, nos referidos anos ${ }^{22}$. Tal percentagem é bastante significativa e é provavelmente o maior levantamento já realizado e divulgado, até o momento, com o objetivo de constatar a frequência de abscessos hepáticos em bovinos de corte brasileiros. Dois trabalhos nacionais se destacam nesse que- 
Tabela 3 - Frequência global de abscessos hepáticos nos bovinos abatidos nos anos estudados - São Paulo - 2009

\begin{tabular}{cccc}
\hline Ano & $\begin{array}{c}\text { Total de bovinos } \\
\text { abatidos }\end{array}$ & $\begin{array}{c}\text { Número de } \\
\text { abscessos hepáticos }\end{array}$ & $\begin{array}{c}\text { Percentual } \\
(\%)\end{array}$ \\
\hline $\mathbf{2 0 0 2}$ & 292.902 & 5.772 & $1,97^{\mathrm{a}}$ \\
$\mathbf{2 0 0 3}$ & 306.362 & 6.394 & $2,08^{\mathrm{a}}$ \\
$\mathbf{2 0 0 4}$ & 337.156 & 5.459 & $1,62^{\mathrm{b}}$ \\
$\mathbf{2 0 0 5}$ & 317.007 & 3.864 & $1,22^{\mathrm{c}}$ \\
$\mathbf{2 0 0 6}$ & 315.394 & 3.727 & $1,18^{\mathrm{d}}$ \\
\hline
\end{tabular}

Letras diversas entre as linhas diferem entre si $(\mathrm{P}<0,0001)$

Tabela 4 - Frequência de abscessos hepáticos em bovinos, machos e fêmeas, independente do tipo de terminações e abatidos no decorrer do estudo - São Paulo - 2009

\begin{tabular}{ccccccc}
\hline \multirow{2}{*}{ Tipo de terminação } & \multicolumn{2}{c}{ Bovinos abatidos } & \multicolumn{2}{c}{ Abscesso hepático } & \multicolumn{2}{c}{ Percentual } \\
\cline { 2 - 7 } & Machos (n) & Fêmeas (n) & Machos (n) & Fêmeas (n) & Machos (\%) & Fêmeas (\%) \\
Extensiva & 981.898 & 184.244 & 12.022 & 2.942 & $1,22^{\mathrm{b}}$ & $1,59^{\mathrm{a}}$ \\
Confinada & 350.830 & 51.849 & 8.820 & 1.432 & $2,51^{\mathrm{b}}$ & $2,76^{\mathrm{a}}$ \\
\hline Total & 1.333 .007 & 235.814 & 20.844 & 4.372 & $1,56^{\mathrm{b}}$ & $1,85^{\mathrm{a}}$ \\
\hline
\end{tabular}

Letras diversas minúsculas entre as colunas e maiúsculas entre as linhas diferem entre si $(\mathrm{P}<0,0001)$

Tabela 5 - Frequência de abscessos hepáticos em bovinos submetidos às diferentes terminações e abatidos no decorrer do estudo e fator de risco (FR) - São Paulo - 2009

\begin{tabular}{|c|c|c|c|c|c|c|c|}
\hline \multirow{2}{*}{ Estado } & \multicolumn{2}{|c|}{ Bovinos abatidos } & \multicolumn{2}{|c|}{ Abscesso hepático } & \multicolumn{2}{|c|}{ Porcentagem } & \multirow{2}{*}{$\begin{array}{l}\text { Fator de risco } \\
\text { (FR) }\end{array}$} \\
\hline & $\underset{(n)}{\text { Confinado }}$ & $\begin{array}{l}\text { Extensivo } \\
\text { (n) }\end{array}$ & $\underset{(n)}{\text { Confinado }}$ & $\begin{array}{l}\text { Extensivo } \\
\text { (n) }\end{array}$ & $\begin{array}{c}\text { Confinado } \\
(\%)\end{array}$ & $\begin{array}{c}\text { Extensivo } \\
(\%)\end{array}$ & \\
\hline SP & 196.951 & 447.887 & 6.622 & 6.261 & $3,36^{\mathrm{aC}}$ & $1,40^{\mathrm{bB}}$ & 2,45 \\
\hline MS & 101.842 & 586.508 & 1.502 & 5.748 & $1,47^{\mathrm{aE}}$ & $0,98^{\mathrm{bD}}$ & 1,52 \\
\hline PR & 31.293 & 68.717 & 1.545 & 1.657 & $4,94^{\mathrm{aA}}$ & $2,41^{\mathrm{bA}}$ & 2,10 \\
\hline GO & 10.104 & 81.980 & 314 & 877 & $3,10^{\mathrm{aD}}$ & $1,07^{\mathrm{bC}}$ & 2,97 \\
\hline MT & 1.052 & 25.179 & 16 & 159 & $1,52^{\mathrm{aE}}$ & $0,63^{\mathrm{bE}}$ & 2,43 \\
\hline MG & 5.111 & 12.197 & 210 & 165 & $4,11^{\mathrm{aB}}$ & $1,35^{\mathrm{bBC}}$ & 3,12 \\
\hline
\end{tabular}

Letras diferentes minúsculas entre as colunas e maiúsculas entre as linhas diferem entre si $(\mathrm{P}<0,0001)$

sito. No primeiro deles, acompanharam tal frequência global em 607.346 bovinos, apenas mencionando que os animais eram provenientes de quatro Estados da Federação (Minas Gerais, Goiás, Mato Grosso e Mato Grosso do Sul), e abatidos em frigoríficos localizados em 11 cidades mineiras ${ }^{2}$. Num trabalho posterior, realizou-se idêntico trabalho com 155.651 bovinos abatidos em único frigorífico na cidade de Uberlândia, sem especificar a procedência do gado ${ }^{18}$. O presente artigo além avaliar a frequência de abscessos em bovinos em cada um dos seis Estados da Federação (São Paulo, Mato Grosso do Sul, Mato Grosso, Goiás, 
Minas Gerais e Paraná), também analisou a influência sexual, e tipo de terminação sobre este achado necroscópico, variáveis não contempladas nos dois outros levantamentos supracitados.

A frequência de condenação global de fígado (Tabela 1), independente ao tipo de terminação, foi de $4,37 \%$, bastante inferior à descrita na literatura, na qual encontraram $8,3 \%$ e $16,52 \%{ }^{2,3}$. Contribuem para esta diferença os trabalhos encontrados de alta frequência de teleangiectasia (2,1\% X 1,67\%), abscessos $(2,02 \%$ X $1,60 \%)$ e hidatidose $(0,38 \%$ X $0,07 \%)$ e de fasciolose $(3,06 \% \text { X 0,007\% })^{2,3}$.

Como era de se esperar, a alteração hepática significativamente mais encontrada (Tabela 1) no presente trabalho foi a teleangiectasia $(1,67 \%)$, com um percentual ligeiramente superior aos abscessos hepáticos (1,60\%). Ambas as alterações somadas representam $75,1 \%$ das causas dos fígados rejeitados para consumo humano.

Uma menor frequência de teleangiectasia foi achada no presente estudo em bovinos terminados em sistema de confinamento que os mantidos extensivamente (Tabela 2), diferentemente dos dados encontrados na literatura ${ }^{17}$. O motivo dessa influência dietética é completamente ignorado e sem uma relação causa-efeito aparente, visto que a patogenia da teleangiectasia continua ainda desconhecida.

A perihepatite aparece como a $3^{\text {a }}$ principal causa de condenação semelhante a outros estudos (Tabela 2$)^{2}$.

Ficou nítido no presente trabalho que bovinos terminados em confinamento apresentaram maior frequência de abscessos que os mantidos apenas em pastoreio $(2,54 \% \mathrm{X} 1,28 \%)$, determinando um aumento no valor do fator de risco na ordem de 2,01 vezes. $\mathrm{Na}$ literatura consultada não foram encontrados dados avaliando esse fator de risco, comparando-se os tipos de terminação. Embora os presentes resultados fossem coerentes, esperava-se que bovinos confinados apresentassem um fator de risco maior ainda do que o encontrado, indicando duas possibilidades, ou que nosso tipo de terminação não é agressivo o suficiente para gerar uma reação em cascata culminando com abscessos hepáticos, ou que a frequência deste problema hepático em bovinos criados extensivamente é alta demais.

A incidência média de abscessos hepáticos em bovinos criados em confinamentos americanos varia de $12 \%$ a $32 \%$. Tal dado revela que a frequência de abscessos em bovinos confinados em nosso meio é muito mais baixa $(2,54 \%)$ do que nos Estados Unidos. Provavelmente, isto é devido ao tipo de manejo alimentar praticado nos dois diferentes países durante o confinamento. Nos Estados Unidos, devido a um problema de manejo e custos, a maioria dos confinamentos adota uma dieta com altíssima quantidade de grãos (entre $80 \%$ a $90 \%$ de grãos, com destaque para milho floculado, de fácil fermentação ruminal) e baixo teor de volumosos, o que aumenta a chance de ocorrer quadros de ruminite ${ }^{15,23,24}$. No Brasil, embora faltem levantamentos, a maioria dos confinamentos emprega percentuais de concentrados (a base de milho inteiro, ou triturado, ligeiramente menos fermentados no rúmen) inferiores a $70 \%$ da dieta ${ }^{25}$.

Outro fato que talvez explique a baixa frequência de abscessos em nossos bovinos confinados, em relação aos americanos é o tempo de permanência em confinamento. A maioria dos abscessos surge nos últimos 60 dias do período de confinamento estadunidense. Além do mais, constatou-se que a frequência de abscessos hepáticos em bovinos, não medicados com antibióticos preventivos, era menos que $5 \%$ nos primeiros meses de confinamento ${ }^{12}$, explicando em parte o porquê do baixo encontro dessa enfermidade em bovinos avaliados no presente estudo.

Surpreendentemente, a frequência de abscessos hepáticos em bovinos não confinados foi relativamente alta em nosso meio (1,28\%). Segundo vários autores as principais origens de abscessos após os quadros de ruminite, são os consequentes aos casos de infecções umbilicais contraídas logo após o nascimento ${ }^{26,27}$. Em- 
bora não exista um trabalho nacional estudando a incidência de enfermidades infecciosas que acometem o umbigo de bezerros neonatos de corte no Brasil, sabe-se que a frequência dessas onfalopatias é alta e considerável em fazendas com problemas de manejo higiênico-sanitários com estes animais ${ }^{28}$. Também podem ocorrer surtos de abscessos hepáticos em bovinos que contraíram infecções umbilicais ${ }^{27}$. Mesmo assim, é pouco provável que apenas as onfalopatias, contraídas nos primeiros dias de vida, sejam responsáveis por uma alta frequência de abscessos hepáticos como a constatada no presente trabalho. Muitos pequenos abscessos hepáticos contraídos durante os primórdios da vida, ou no início do período de confinamento, são devidamente reabsorvidos, simplesmente deixando de serem diagnosticados ou se transformando numa pequena cicatriz. Os resultados obtidos no levantamento retrospectivo poderão trazer alguma luz para explicar a frequência relativamente alta em bovinos não confinados, os quais serão discutidos a seguir, em especial quanto ao surgimento de abscesso hepáticos decorrentes dos quadros de broncopneumonias ${ }^{12}$.

Outro dado importante que este trabalho levantou é que existiu entre as populações de bovinos estudados, tanto terminados em confinamento com em pastoreio, uma tendência de diminuição de frequência de abscessos no decorrer dos anos de 2002 a 2006. O motivo é completamente ignorado, mas especula-se que na terminação intensiva algumas condições podem ter interferido no surgimento desses resultados. É possível que nos anos de 2005 e 2006, em que o preço da arroba do boi gordo caiu significativamente em relação aos anos anteriores estudados, acompanhado de elevação concomitante do preço de custo de produção, tenha levado muitos confinadores a reduzir o oferecimento de concentrados ricos em energia, substituindo estes por volumosos, com intuito de diminuir os custos de produção ${ }^{28}$. Outro fator que pode ter influenciado é a inclusão em dietas e bovinos confina- dos de virginiamicina e tilosina, antibióticos estes que reconhecidamente são empregados para a prevenção dos citados abscessos.

Em relação à tendência de redução de abscessos nos bovinos terminados extensivamente especula-se também que melhoras no manejo geral do gado em questão tenham diminuído o risco desta infecção hepática.

Em todas as situações sob regime de terminação extensiva, as fêmeas obtiveram maior percentual global (1,59\% X 1,22\%) de abscessos hepáticos em relação aos machos. Também foi encontrada frequência maior nas fêmeas quando submetidas à terminação em confinamento $(2,76 \%$ X 2,51\%). Contudo, quando foi calculado o fator de risco da influência sexual de surgimento de abscessos hepáticos, independente do tipo de terminação, não foram constatadas diferenças entre os sexos $(F R=0,84)$. Tais resultados contrariam a literatura clássica que indica que machos são mais predispostos que as fêmeas a apresentarem abscessos hepáticos por ingerirem maior quantidade de matéria seca que as fêmeas e que estas são confinadas por um tempo mais breve ${ }^{10,12,27}$. São desconhecidos os motivos dos presentes resultados, mas especula-se que provavelmente as fêmeas nacionais apresentem outras enfermidades internas que de alguma forma predisponha o surgimento de abscessos. Mais estudos são necessários para elucidar tal suposição.

\section{Conclusão}

Concluiu-se que os abscessos hepáticos são o segundo principal motivo de descarte de fígados, após as telengectasias; a frequência desses abscessos é maior entre os bovinos terminados em regime de confinamento que os criados extensivamente; as fêmeas, tanto terminadas extensivamente como em confinamento, apresentaram maior frequência de abscesso que os machos e a frequência de abscessos foi maior em bovinos criados no Estado do Paraná em ambos os regimes de terminação. 


\section{Referências}

1.KRAUSE, K. M.; OETZEL, G. R. Understanding and preventing subacute ruminal acidosis in dairy herds: a review. Animal Feed Science and Technology, v.126, n. 3/4, p. 215236, 2006 .

2. BAPTISTA, F.; MOREIRA, E. C. Causas de condenação de fígados bovinos em frigorífico de Minas Gerais e perdas econômicas associadas. Revista Higiene Alimentar, v. 13, n. 62, p. 22-28, 1999.

3. MENDES, R. E.; PILATI, C. Estudo morfológico de fígado de bovinos abatidos em frigorífico industriais sob inspeção estadual no Oeste e no Planalto de Santa Catarina, Brasil Ciência Rural, Santa Maria, v. 37, n. 6, p. 1728-1734, 2007.

4. JENSEN, R.; CONNELL, W. E.; DEEM, A. W. Rumenitis and relation to rate of change of ration and the proportion of concentrate in the ration of cattle. America Journal Veterinary Research, v. 15, n. 56, p. 425-428, 1954.

5. JENSEN, R.; DEANE, H. N.; COOPER, L. J.; MILLER, V. A.; GRAHAM, W. R. The rumenits-liver abscess complex in beef cattle. America Journal Veterinary Research, v. 15, n. 56, p. 202-216, 1954.

6. JENSEN, R.; FLINT, J. C.; UDALL, R. H.; DEEM, A. W.; SEGER, C. L. Parakeratosis of the rumens of lambs fattened on pelleted feed. America Journal Veterinary Research, v. 19, n. 71, p. 277-282, 1958.

7.BROWN, H.; ELLISTON, N. G.; MCASKILL, J. W. MUENSTER, O. A.; TONKINSON, L. V. Tylosin phosphate (TP) and tylosin urea adduct (TUA) for the prevention of liver abscess, improved weight gains and feed efficiency in feedlot cattle. Journal Animal Science, v. 37, n. 5, p. 1085-1091, 1973.

8. BROWN, H.; BING, R. F.; GRUETER, H. P.; MCASKILL, J. W.; COOLEY, C. O.; RATHMACHER, R. P. Tylosin and chlortetracycline for prevention of liver abscess, improved weight gains and feed efficiency in feedlot cattle. Journal Animal Science, v. 40, n. 2, p. 207-213, 1975.

9. LECHTENBERG， K. F.; NAGARAJA， T. G. Hepatic ultrasonography and blood changes in steers with experimentally induced liver abscess. America Journal Veterinary Research, v. 52, n. 6, p. 803-809, 1991.

10.LECHTENBERG, K. F.; NAGARAJA, T. G.; CHENGAPPA, M. M. Antimicrobial susceptibility of Fusobacterium necrophorum isolated from bovine hepatic abscess. America Journal Veterinary Research, v. 59, n. 1, p. 44-47, 1998.

11.NAGARAJA, T. G.; CHENGAPPA, M. M. Liver abscess in feedlot: a review. Journal Animal Science, v. 76, n. 1, p. 287298, 1998 .

12.NAGARAJA, T. G.; LECHTENBERG, K. F. Liver abscess in feedlot cattle. Veterinary Clinics Food Animal, v. 23, n. 2, p. 351-369, 2007.

13. FOSTER, L.; WOOD, W. R. Liver losses in finishing cattle.
Lincoln: Nebraska beef cattle report, University of Nebraska, 1970. p. 2-4.

14.LECHTENBERG, K. F.; NAGARAJA, T. G.; LEIPOLD, H. W.; CHENGAPPA, M. M. Bacteriologic and histologic studies of hepatic abscess in cattle. America Veterinary Research, v. 49, n. 1 , p. $58-62,1988$.

15. BRINK, D. R.; LOWRY, S. R.; STOCK, R. A.; PARROTT, J. C. Severity of liver abscess and efficiency IF feed utilization of feedlot cattle. Journal Animal Science, v. 68, n. 5, p. 1201-1207, 1990.

16. NAGARAJA, T. G.; CHENGAPPA, M. M. Liver abscess in feedlot: a review. Journal Animal Science, v. 76, p. 287-298, 1998.

17. ROBERTS, J. L. The prevalence and economic significance of liver disorders and contamination in grain-fed and grass-fed cattle. Australian Veterinary Journal, v. 59, n. 5, p. 129-132, 1982.

18. ALMEIDA, L. P.; REIS, D. O.; CARRIJO, K. F. Abscessos hepáticos como causa de condenações em bovinos de abate. Revista Higiene Alimentar, v. 19, n. 134, p. 51-53, 2005.

19.SAMPAIO, I. B. M. Estatística aplicada à experimentação animal. 2. ed. Belo Horizonte: Fundação de Estudo e Pesquisa em Medicina Veterinária e Zootecnia, 2002, 265 p.

20.LATORRE, M. R. D. O. Medidas de risco e regressão logística. In: MASSAD, E.; MENEZES, R. X.; SILVEIRA, P. S. P.; ORTEGA, N. R. S. Métodos quantitativos em medicina. Barueri: Manole, 2004, cap. 16, p. 337-350.

21. MINITAB. The student edition of MINITAB Statistical software for education. Release 13.0 User's Manual. New York: Addison - Wesley, 2000. CD-ROM.

22. PITOMBO, L. H. Corte em números. Anuário Revista DBO, v. 26, n. 315, p.7-29, 2007.

23. SMITH, R. A. Impact of disease on feedlot performance: a review. Journal Animal Science, v.76, p. 272-274, 1998.

24.SANTOS, F. A. P.; MOSCARDINI, M. C. Substituição de fontes de amido por subprodutos ricos em pectina ou fibra de alta digestibilidade na ração de bovinos confinados. In: SIMPÓSIO DE NUTRIÇÃO DE RUMINANTES, 3.; BRAZILIAN RUMINANT NUTRITION CONFERENCE RÚMEN HEALTH, 1., 2007, Botucatu. [Anais...] São Paulo: UNESP, 2007. p. 35-52.

25.FRANCO, M. Alto grão x alto volumoso. Revista DBO, v. 25, n. 308 , p. 54-58, 2006. (Matéria de capa)

26. RADOSTITS, O. M.; GAY, C. C.; BLOOD, D. C.; HINCHCLIFF, K. W.; CONSTABLE, P. D. Veterinary medicine. 10. ed. Philadelphia: Saunders, p. 935-946, 2007.

27.FIGUEIREDO, L. J. C. Onfalopatia de bezerro. Salvador: Editora da Universidade Federal da Bahia, 1999. 94 p.

28.CEPEA, 2009. Disponível em: <www.cepea.esalq.usp.br> Acessado em 11 fev. 2009. 